\title{
Valvular heart disease as a possible predictor of trastuzumab-induced cardiotoxicity in patients with breast cancer
}

\author{
AKIHIKO SATO ${ }^{1}$, AKIOMI YOSHIHISA ${ }^{1}$, MAKIKO MIYATA-TATSUMI ${ }^{1}$, MASAYOSHI OIKAWA ${ }^{1}$, \\ ATSUSHI KOBAYASHI ${ }^{1}$, TAKAFUMI ISHIDA ${ }^{1}$, TOHRU OHTAKE $^{2}$ and YASUCHIKA TAKEISHI ${ }^{1}$ \\ Departments of ${ }^{1}$ Cardiovascular Medicine and ${ }^{2}$ Breast Surgery, Fukushima Medical University, \\ Fukushima, Fukushima 960-1295, Japan
}

Received March 1, 2018; Accepted October 2, 2018

DOI: $10.3892 / \mathrm{mco} .2018 .1764$

\begin{abstract}
Although the use of trastuzumab has been reported to improve overall survival in patients with HER2-positive breast cancer, there is increasing concern about the adverse effects of trastuzumab-induced cardiotoxicity (TIC). The aim of the present study was to investigate the predictor of TIC and to consider appropriate management for such patients. The present study breast cancer 119 patients with breast cancer who had been treated with trastuzumab. Patients were referred to our department for cardiac function screening. The patients' baseline characteristics, echocardiographic data, presence of trastuzumab-induced cardiotoxicity (TIC) and all-cause mortality were investigated. TIC was defined as a manifestation of overt heart failure or $\geq 10 \%$ reduction of left ventricular ejection fraction (LVEF) from baseline to an LVEF $<55 \%$ in asymptomatic patients. During the follow-up period (mean, 1,410 days), symptomatic heart failure occurred in 2 out of 119 patients (1.6\%), 11 patients $(9.2 \%)$ had asymptomatic impairment of cardiac function that was ameliorated by discontinuing trastuzumab and 20 patients (16.8\%) succumbed to cancer-associated fatality. In the logistic regression analysis, only the presence of valvular heart disease at the baseline was indicated to be a predictor of TIC. There was no other predictor for TIC, including baseline characteristics, other therapies and echocardiographic parameters. In addition, impairment of cardiac function was ameliorated by discontinuing trastuzumab. TIC occurred in $\sim 10 \%$ of the patients treated with trastuzumab. Only the presence of valvular heart disease seems to be associated with occurrence of TIC, with no other specific predictor of TIC demonstrated in the present study. The present data suggests the importance
\end{abstract}

Correspondence to: Professor Akiomi Yoshihisa, Department of Cardiovascular Medicine, Fukushima Medical University, 1 Hikarigaoka, Fukushima, Fukushima 960-1295, Japan

E-mail: yoshihis@fmu.ac.jp

Key words: cardio-oncology, trastuzumab, echocardiography, left ventricular ejection fraction, valvular heart disease of regular monitoring of cardiac function, and that presence of valvular heart disease may be a possible predictor of TIC.

\section{Introduction}

Cancer is a major public health problem worldwide, and there are approximately 15.5 million patients with a history of cancer in the United States (1); thus, there is great public concern about cancer therapy. The development of anticancer drugs, with the shift from traditional chemotherapies to molecular-targeted therapies in the last decade, have dramatically improved the clinical outcome of cancer patients, and have resulted in prolonging the life span of these patients (2). Traditional chemotherapies target the cell cycle of malignant cells as well as that of normal cells, resulting in adverse effects on cytotoxicity such as alopecia, gastrointestinal toxicity, myelotoxicity and cardiotoxicity. In contrast, molecular-targeted therapies with monoclonal antibodies specifically target malignant cells. The specificity of molecular targeted therapies for malignant cells is the reduction of side effects as well as more effective treatment of various kinds of cancer, when compared with traditional standard chemotherapies. However, adverse effects on cardiac function (i.e., cardiotoxicity) for several molecular target agents have been reported $(3,4)$.

Trastuzumab, a monoclonal antibody administered for breast cancer patients, interferes with human epidermal growth factor receptor 2 (HER2), which is a kind of trans-membrane tyrosine kinase that regulates cell growth, cell survival, adhesion, migration, and differentiation $(5,6)$. HER2 is overexpressed in approximately $25 \%$ of breast cancer cases, and HER2-positive breast cancer tends to have a high risk of metastasis, resistance for anticancer drugs, and recurrence, because overactivation of HER2 signaling both promotes cell proliferation and inhibits cell death (7). Trastuzumab significantly improves overall survival in patients with HER2-positive breast cancer by blocking HER2 signaling (8). Although HER2 signaling also plays an essential role in the maintenance of cardiomyocyte function $(9,10)$, there is recent increasing concern about the adverse effects of trastuzumab-induced cardiotoxicity (TIC), which is occasionally life-threatening. Unfortunately, the risk factors and predictors of TIC in breast cancer patients have yet to be fully elucidated $(3,11,12)$. 
The aims of this study were to investigate the predictors of TIC, and to consider appropriate management for such patients.

\section{Patients and methods}

Study design and subjects. This was a retrospective study to investigate the prevalence of TIC and its predictors. The clinical data of consecutive 450 breast cancer patients, who had been referred to our department to undergo echocardiography before chemotherapy and/or operation between 2003 and 2015, were investigated, including baseline characteristics, echocardiographic parameters, and occurrence of TIC or all-cause death. Trastuzumab was administered as a loading dose of $8 \mathrm{mg} / \mathrm{kg}$, followed by $6 \mathrm{mg} / \mathrm{kg}$ every three weeks (13). We evaluated several co-morbidities and past histories in the medical records that may be associated with occurrence of TIC and mortality, such as the presence and/or past history of hypertension, dyslipidemia, diabetes mellitus, chronic kidney disease, ischemic heart disease, arrhythmia, valvular heart disease (VHD) and heart failure, as well as past treatment of anthracycline or radiation. Echocardiography was performed by experienced echocardiographers using standard techniques. The definition of TIC was: i) Manifestation of decompensated heart failure, based on the Framingham criteria (14); or ii) $<55 \%$ of left ventricular ejection fraction (LVEF) and a reduction of LVEF of $>10 \%$ from baseline to after trastuzumab treatment in asymptomatic patients (15). Out of 450 patients, 119 patients received trastuzumab therapy. We divided the 119 patients into two groups based on the presence or absence of TIC (TIC group, $\mathrm{n}=13$ and non-TIC group, $\mathrm{n}=106$ ).

Statistical analysis. Normally distributed data are presented as mean $\pm \mathrm{SD}$. The baseline characteristics between the two groups were compared using the independent Student's t-test for parametric variables, and the Chi-square test was used for categorical variables. Comparisons of data at baseline and after trastuzumab treatment were analyzed using the paired Student's t-test. We performed logistic regression analysis allowing for interaction between the onset of TIC and each possible confounding factor. These analyses were performed using a statistical software package (SPSS version 24.0; IBM, Armonk, NY, USA), and a P-value of $<0.05$ was considered statistically significant. The study protocol was approved by the ethics committee of Fukushima Medical University.

\section{Results}

The baseline characteristics of the 119 patients are presented in Table I. There were no significant differences, except for the presence of VHD, in the baseline characteristics and echocardiographic parameters between the TIC and non-TIC groups before initiation of trastuzumab (Table I). The details of VHD were as follows: i) Moderate mitral regurgitation, $\mathrm{n}=4$; ii) moderate aortic regurgitation, $\mathrm{n}=3$; and iii) moderate tricuspid regurgitation, $\mathrm{n}=5$. Slight-to-mild VHD was not considered as significant VHD, and there were no patients with severe VHD in the present study. Seventy-two patients $(60.5 \%)$ received a combination therapy with anthracycline,
$38(31.9 \%)$ received radiation therapy, and $27(22.6 \%)$ received both therapies. In the follow-up period (mean, 1,410 days), symptomatic heart failure occurred in two patients (1.6\%), and 11 patients $(9.2 \%)$ had asymptomatic impairment of cardiac function, as determined by echocardiography. The impairment of cardiac function was ameliorated by discontinuing trastuzumab. The time courses of LVEF in each patient with TIC are presented in Fig. 1. The LVEF in all TIC patients was recovered after discontinuing trastuzumab. Twenty patients (16.8\%) died due to cancer-related causes, and no patients died of cardiac death. Within 3 to 6 months of initiation of treatment with trastuzumab, the TIC group showed increased volumes of the left ventricle and atrium, and decreases of the fractional shortening, LVEF and E'. In the present logistic regression analysis, only the presence of VHD at baseline was a predictor of TIC, and there was no other predictor for TIC, including baseline characteristics, other therapies and baseline echocardiographic parameters, in the present study (Table II).

\section{Discussion}

Although trastuzumab improves the prognosis of patients with HER2-positive breast cancer, TIC is an important issue. The exact mechanism of TIC is still remains unknown; however, several mechanisms have been suspected $(16,17)$. HER2 conditional knockout mice generally develop severe dilated cardiomyopathy (16). Thus, HER2, the preferred dimerization partner for all other ErbB receptors, particularly ErbB4 in cardiomyocytes, is thought to have a variety of roles in the normal physiology of the heart, including morphology, hypertrophic growth, excitation-contraction coupling, and survival (16). Neuregulin activates both ErbB2 and ErbB4 receptor tyrosine kinase activity and promotes growth, myofilament organization, and survival of isolated cardiac myocytes (17). By circulating regulating reactive oxygen species-induced cardiomyocyte apoptosis, inducing cell-cycle activity, neuregulin normally protects cardiomyocytes against stress, promoting regeneration and improving cardiac function and survival (16). Inhibition of HER2 signaling with trastuzumab antagonizes the effects of neuregulin (16).

In the present retrospective study, asymptomatic TIC occurred in $9.2 \%$ of the study population, and symptomatic HF occurred in $1.6 \%$. These results are concordant with those of a recent large retrospective cohort study by Bowles et al, of 12,500 women who had been diagnosed with breast cancer between 1999 and 2007 in the United states. That study reported a TIC occurrence of $9.8 \%$ of the study population, and symptomatic HF occurrence in $2.7 \%$ (18). In addition, LVEF recovery was observed in all patients with TIC after the discontinuation of trastuzumab in the current study, which is consistent with the findings of Bowles's study. Compared with no chemotherapy, the incidence of TIC in Bowles's study was higher in patients treated with anthracycline alone [adjusted hazard ratio $(\mathrm{HR})=1.40,95 \%$ confidence interval $(\mathrm{CI})$ : 1.11-1.76]. Although the increased risk was similar to those of other chemotherapy (adjusted HR=1.49, 95\% CI: 1.25-1.77), the risk was highly increased in patients treated with trastuzumab alone (adjusted HR=4.12, 95\% CI: 2.30-7.42) or anthracycline plus trastuzumab (adjusted HR= 7.19, 95\% CI: 5.00-10.35) (18). Data from clinical trials have indicated that anthracycline 
Table I. Patient demographics.

\begin{tabular}{|c|c|c|c|}
\hline & TIC (-) $(\mathrm{n}=106)$ & $\mathrm{TIC}(+)(\mathrm{n}=13)$ & P-value \\
\hline \multicolumn{4}{|l|}{ Demographics } \\
\hline Age (years) & $56.7 \pm 11.1$ & $51.9 \pm 9.1$ & 0.093 \\
\hline Female sex $(\mathrm{n}, \%)$ & $106(100)$ & $13(100)$ & 0.806 \\
\hline Body mass index $\left(\mathrm{kg} / \mathrm{cm}^{2}\right)$ & $22.5 \pm 3.9$ & $20.1 \pm 2.7$ & 0.062 \\
\hline \multicolumn{4}{|l|}{ Co-morbidity } \\
\hline Hypertension (n, \%) & $22(20.8)$ & $0(0)$ & 0.174 \\
\hline Dyslipidemia (n, \%) & $22(20.8)$ & $2(15.4)$ & 0.841 \\
\hline Diabetes mellitus (n, \%) & $10(9.4)$ & $1(7.7)$ & 0.919 \\
\hline Chronic kidney disease (n, \%) & $7(5.6)$ & $1(7.7)$ & 0.879 \\
\hline Ischemic heart disease & $2(1.9)$ & 0 & 0.617 \\
\hline Arrhythmia & $5(4.7)$ & $1(7.7)$ & 0.644 \\
\hline Valvular heart disease & $4(3.8)$ & $3(23.1)$ & 0.047 \\
\hline \multirow{2}{*}{ MR/AR/TR } & $1(0.9) / 2$ & $3(23.1) / 1$ & \\
\hline & $(1.9) / 3(2.8)$ & $(7.7) / 2(15.4)$ & \\
\hline Heart failure & $1(0.9)$ & $1(7.7)$ & 0.074 \\
\hline \multicolumn{4}{|l|}{ Laboratory data } \\
\hline Hemoglobin $(\mathrm{g} / \mathrm{dl})$ & $11.7 \pm 1.5$ & $11.4 \pm 1.4$ & 0.514 \\
\hline Creatinine (mg/dl) & $0.59 \pm 0.12$ & $0.65 \pm 0.14$ & 0.250 \\
\hline Estimated GFR $\left(\mathrm{ml} / \mathrm{min} / \mathrm{m}^{2}\right)$ & $82.9 \pm 20.2$ & $87.5 \pm 25.2$ & 0.461 \\
\hline \multicolumn{4}{|l|}{ Combination therapy } \\
\hline Anthracycline (n, \%) & $62(58.5)$ & $10(76.9)$ & 0.163 \\
\hline Radiation therapy (n, \%) & $36(34.0)$ & $2(15.4)$ & 0.221 \\
\hline Anthracycline and radiation therapy $(\mathrm{n}, \%)$ & $26(24.5)$ & $1(7.7)$ & 0.293 \\
\hline \multicolumn{4}{|c|}{ Echocardiographic parameters (Baseline and after trastuzumab) } \\
\hline IVS (mm): Baseline & $9.1 \pm 1.3$ & $9.8 \pm 0.9$ & 0.098 \\
\hline After & $9.4 \pm 3.3$ & $9.8 \pm 1.9$ & 0.627 \\
\hline PW (mm): Baseline & $9.6 \pm 3.1$ & $9.7 \pm 0.6$ & 0.805 \\
\hline After & $9.3 \pm 1.5$ & $9.9 \pm 1.4$ & 0.270 \\
\hline LVDd (mm): Baseline & $42.2 \pm 4.9$ & $43.5 \pm 3.6$ & 0.360 \\
\hline After & $43.1 \pm 4.9$ & $48.1 \pm 4.1^{\mathrm{a}}$ & 0.004 \\
\hline LVDs (mm): Baseline & $26.1 \pm 4.9$ & $28.0 \pm 3.8$ & 0.146 \\
\hline After & $26.7 \pm 4.4$ & $35.5 \pm 5.6^{\mathrm{a}}$ & $<0.001$ \\
\hline FS (\%): Baseline & $38.0 \pm 8.1$ & $33.5 \pm 4.7$ & 0.196 \\
\hline After & $37.5 \pm 7.3$ & $23.6 \pm 8.7^{\mathrm{a}}$ & $<0.001$ \\
\hline LA diameter (mm): Baseline & $31.2 \pm 5.3$ & $31.5 \pm 7.6$ & 0.862 \\
\hline After & $31.2 \pm 6.5$ & $34.2 \pm 5.2$ & 0.164 \\
\hline LA volume (ml): Baseline & $35.7 \pm 16.1$ & $37.3 \pm 19.9$ & 0.783 \\
\hline After & $35.5 \pm 17.3$ & $54.8 \pm 17.6^{\mathrm{a}}$ & 0.005 \\
\hline LVEDV (ml): Baseline & $63.4 \pm 19.6$ & $68.2 \pm 11.0$ & 0.385 \\
\hline After & $65.0 \pm 21.2$ & $90.4 \pm 17.1^{\mathrm{b}}$ & 0.001 \\
\hline LVESV (ml): Baseline & $22.7 \pm 8.3$ & $26.1 \pm 4.9$ & 0.149 \\
\hline After & $24.0 \pm 10.1$ & $48.6 \pm 15.5^{\mathrm{b}}$ & $<0.001$ \\
\hline LVEF (\%): Baseline & $64.3 \pm 5.7$ & $61.9 \pm 4.7$ & 0.147 \\
\hline After & $64.2 \pm 5.5$ & $46.9 \pm 8.0^{\mathrm{b}}$ & $<0.001$ \\
\hline TRPG (mmHg): Baseline & $17.3 \pm 6.6$ & $18.3 \pm 6.9$ & 0.663 \\
\hline After & $19.8 \pm 7.3$ & $22.8 \pm 9.3$ & 0.228 \\
\hline $\mathrm{E}(\mathrm{m} / \mathrm{sec})$ : Baseline & $0.68 \pm 0.16$ & $1.1 \pm 1.6$ & 0.321 \\
\hline After & $0.71 \pm 0.17$ & $0.82 \pm 0.33$ & 0.333 \\
\hline A (m/sec): Baseline & $0.73 \pm 0.18$ & $0.65 \pm 0.16$ & 0.086 \\
\hline After & $0.73 \pm 0.16$ & $0.66 \pm 0.19$ & 0.235 \\
\hline $\mathrm{E}^{\prime}(\mathrm{cm} / \mathrm{sec})$ : Baseline & $8.7 \pm 2.9$ & $8.2 \pm 1.6$ & 0.564 \\
\hline After & $9.3 \pm 4.3$ & $5.6 \pm 3.9^{\mathrm{a}}$ & 0.030 \\
\hline
\end{tabular}


Table I. Continued.

\begin{tabular}{|c|c|c|c|}
\hline & TIC (-) $(n=106)$ & $\mathrm{TIC}(+)(n=13)$ & P-value \\
\hline IVC (mm): Baseline & $11.8 \pm 2.8$ & $12.3 \pm 2.6$ & 0.570 \\
\hline After & $12.3 \pm 3.3$ & $14.8 \pm 4.0^{\mathrm{a}}$ & 0.047 \\
\hline
\end{tabular}

\section{Time course of LVEF in TIC group}

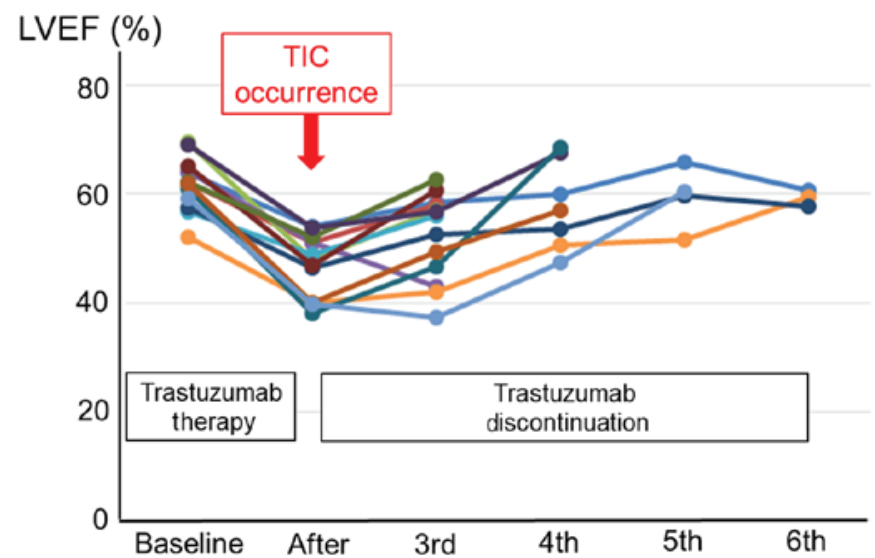

Figure 1. Time course of LVEF in the TIC group. Change of LVEF in each patients of TIC group are presented. The LVEF in all patients with TIC was recovered after discontinuing trastuzumab. LVEF, left ventricular ejection fraction; TIC, trastuzumab-induced cardiotoxicity.

is associated with an approximate $2 \%$ increase (19) in heart failure and/or cardiomyopathy incidence, and anthracycline followed by trastuzumab is associated with an approximate $4 \%$ increase (20). Although several other clinical studies have investigated the risk factors or predictors for TIC $(12,21)$, said predictors TIC have yet to be fully elucidated, and regular cardiac function monitoring has been recommended (22).

Recently, a position paper by the European Society of Cardiology reported that: i) Age, high body mass index $>30 \mathrm{~kg} / \mathrm{m}^{2}$, hypertension, previous or concomitant anthracycline treatment, previous radiation therapy, previous LV dysfunction are suggested factors associated with TIC (3); and that ii) cumulative dose, female sex, age, chronic kidney disease, hypertension, previous radiation therapy, concomitant chemotherapy, pre-existing cardiac disease associated with increased wall stress are suggested factors that are all associated with risk of cardiotoxicity following anthracycline treatment (3).

In contrast to previous data $(3,11,12,18,21)$, in the present study, only presence of VHD was a novel predictor of TIC, but not age, body mass index, other cardiac diseases, combined anthracycline therapy or LVEF. We cannot fully explain the reasons for the differences between said reports and the current results; however, the impact of VHD on TIC were considered as follows: First, VHD has not yet been fully focused on in previous studies in TIC $(12,18,21)$. In contrast, the use of trastuzumab may be avoided in patients with past histories of other cardiac diseases, as stipulated in the recent trastuzumab guidelines (23). Second, presence of VHD itself may indicate latent cardiac damage associated with past endocarditis, radiation, chemotherapy and/or secondary to LV dysfunction (3). Radiation causes fibrosis and calcification of the aortic root, aortic valve cusps, mitral valve annulus and base and mid portions of the mitral valve leaflets, sparing the mitral valve tips and commissures $(3,24)$. Third, VHD is associated with increased wall stress, which is a factor of cardiotoxicity $(3,11,12)$. Fourth, mitral regurgitation results in the overestimation of baseline LVEF. Additionally, with respect to concomitant anthracycline therapy, cardiotoxicity with anthracyclines is generally known to be dose-dependent. A cardiotoxicity incidence occurred in $3-5 \%$ of patients administered anthracyclines at a dose of $400 \mathrm{mg} / \mathrm{m}^{2}, 7-26 \%$ at a dose of $550 \mathrm{mg} / \mathrm{m}^{2}$, and $18-48 \%$ at a dose of $700 \mathrm{mg} / \mathrm{m}^{2}$ (3). In our study subjects, 72 patients $(60.5 \%)$ received anthracycline therapy, with a mean dose of $270 \pm 90 \mathrm{mg} / \mathrm{m}^{2}$; hence, the impact of anthracycline on cardiotoxicity seems to be less in the present study than in previous studies $(3,11,12,18,21)$.

Moreover, some studies have reported that cardiac diastolic dysfunction, evaluated by echocardiography before chemotherapy, might also be a predictor for chemotherapy-induced cardiotoxicity (25), since diastolic dysfunction precedes the appearance of systolic dysfunction in patients treated with chemotherapy (26). It has also been reported that the evaluation of cardiac function using modalities other than echocardiography, such as cardiac magnetic resonance imaging, radionuclide imaging, and positron emission tomography/magnetic resonance, may be able to predict TIC (11). On the other hand, the usefulness of cardiac biomarkers, such as troponin I, troponin $\mathrm{T}$, natriuretic peptide, and high-sensitive C-reactive protein, as predictors for TIC has not been proven yet (27), although the elevation of troponin I in patients with TIC has been reported to predict the irreversibility of LVEF decline and a higher incidence of cardiac events (28). Furthermore, baseline clinical characteristics, cardiac functional parameters and biomarkers have not been well evaluated as predictors for TIC.

There are several limitations in the present study. First, this was a retrospective study of a single institution, and the number of study subjects was relatively small. The study population might be insufficient, and thus it is possible that there were additional factors associated with TIC that were not detected. In addition, the small number of subjects may not be enough to fully explain the relationship between VHD with 
Table II. Univariate logistic regression analysis to determine factors related to TIC.

\begin{tabular}{|c|c|c|c|c|}
\hline Factors & $\beta$ coefficient & P-value & OR & $95 \% \mathrm{CI}$ \\
\hline Age & 0.037 & 0.135 & 0.962 & 0.913-1.012 \\
\hline Body mass index & -0.213 & 0.248 & 0.808 & $0.666-0.118$ \\
\hline Hypertension & -18.637 & 0.998 & - & - \\
\hline Dyslipidemia & -0.398 & 0.592 & 0.672 & $0.156-2.883$ \\
\hline Diabetes mellitus & -0.315 & 0.712 & 0.730 & $0.137-3.879$ \\
\hline Chronic kidney disease & -0.064 & 0.884 & 0.938 & $0.398-2.212$ \\
\hline Ischemic heart disease & -19.123 & 0.999 & - & - \\
\hline Arrhythmia & 0.521 & 0.647 & 1.683 & $0.181-15.637$ \\
\hline Valvular heart disease & 2.035 & 0.015 & 7.650 & $1.496-39.114$ \\
\hline Heart failure & 2.169 & 0.134 & 8.750 & $0.514-149.079$ \\
\hline Combination with anthracycline therapy & 0.861 & 0.210 & 2.366 & $0.615-9.096$ \\
\hline Combination with radiation therapy & -1.735 & 0.102 & 0.176 & $0.022-1.413$ \\
\hline Combination with anthracycline and radiation therapy & -19.361 & 0.998 & - & - \\
\hline LVDd (mm) & 0.058 & 0.357 & 1.060 & $0.936-1.200$ \\
\hline LVEF (\%) & -0.078 & 0.149 & 0.925 & $0.832-1.028$ \\
\hline LA diameter (mm) & 0.09 & 0.860 & 1.009 & $0.911-1.119$ \\
\hline E wave $(\mathrm{m} / \mathrm{sec})$ & 0.988 & 0.306 & 2.686 & $0.400-18.017$ \\
\hline A wave (m/sec) & -3.135 & 0.118 & 0.043 & $0.001-2.219$ \\
\hline $\mathrm{E}^{\prime}$ wave $(\mathrm{cm} / \mathrm{sec})$ & -0.076 & 0.560 & 0.927 & $0.718-1.196$ \\
\hline
\end{tabular}

TIC, trastuzumab induced cardiomyopathy; OR, odds ratio; CI, confidence interval; BNP, B-type natriuretic peptide; LVDd, left ventricle diastolic diameter; LVEF, left ventricle ejection fraction; LA, left atrium.

TIC. Although we considered the use of anthracycline, radiation and combination therapies as risk factors of TIC, logistic regression analysis demonstrated that these factors were not associated with TIC in the present study. However, it should be noted that we could not fully deny the respective impacts of these markers on TIC. Another limitation is that we only examined echocardiography in the present study, and cardiac biomarkers and other cardiac imaging were not fully examined. Furthermore, unfortunately, because it is a retrospective study, we could not fully check the amount of trastuzumab dose or doses in each patient. Therefore, our results are preliminary, and further studies with larger populations and multifaceted evaluations using other cardiac imaging and/or biomarkers are needed.

In the current study, we found that TIC occurred in approximately $10 \%$ of all breast cancer patients who received treatment with trastuzumab. The impaired cardiac function was ameliorated by discontinuing trastuzumab. In addition, the presence of valvular heart disease might be a possible predictor of TIC occurrence in breast cancer patients. Our present data suggests the importance of regular monitoring of cardiac function.

\section{Acknowledgements}

Not applicable.

\section{Funding}

No funding was received.

\section{Availability of data and materials}

The datasets used and/or analysed during the current study are available from the corresponding author on reasonable request.

\section{Authors' contributions}

AS and AY drafted the article and designed this study. MMT and MO performed statistical analysis. AK, TI and TO obtained general data. YT designed this study, obtained general data and revised the article critically for important intellectual content. All authors read and approved the final manuscript.

\section{Ethics approval and consent to participate}

The study protocol conforms to the ethical guidelines of the 1975 Declaration of Hlsinki as reflected in a prior approval by the institution's human research committee (Fukushima Medical University). Patient consent was obtained at the time of data collection.

\section{Patient consent for publication}

Not applicable.

\section{Competing interests}

The authors declare that they have no competing interests. 


\section{References}

1. Miller KD, Siegel RL, Lin CC, Mariotto AB, Kramer JL, Rowland JH, Stein KD, Alteri R and Jemal A: Cancer treatment and survivorship statistics, 2016. CA Cancer J Clin 66: 271-289, 2016.

2. Santhosh S, Kumar P, Ramprasad V and Chaudhuri A: Evolution of targeted therapies in cancer: Opportunities and challenges in the clinic. Future Oncol 11: 279-293, 2015.

3. Zamorano JL, Lancellotti P, Rodriguez Muñoz D, Aboyans V, Asteggiano R, Galderisi M, Habib G, Lenihan DJ, Lip GYH, Lyon AR, et al: Group ESCSD. 2016 esc position paper on cancer treatments and cardiovascular toxicity developed under the auspices of the esc committee for practice guidelines: The task force for cancer treatments and cardiovascular toxicity of the european society of cardiology (esc). Eur Heart J 37: 2768-2801, 2016.

4. Lal H, Kolaja KL and Force T: Cancer genetics and the cardiotoxicity of the therapeutics. J Am Coll Cardiol 61: 267-274, 2013

5. Nikolai BC, Lanz RB, York B, Dasgupta S, Mitsiades N, Creighton CJ, Tsimelzon A, Hilsenbeck SG, Lonard DM, Smith CL and O'Malley BW: HER2 signaling drives DNA anabolism and proliferation through SRC-3 phosphorylation and E2F1-regulated genes. Cancer Res 76: 1463-1475, 2016.

6. Hudis CA: Trastuzumab-mechanism of action and use in clinical practice. N Engl J Med 357: 39-51, 2007.

7. Pritchard KI, Shepherd LE, O'Malley FP, Andrulis IL, Tu D, Bramwell VH and Levine MN; National Cancer Institute of Canada Clinical Trials Group: HER2 and responsiveness of breast cancer to adjuvant chemotherapy. N Engl J Med 354: 2103-2111, 2006.

8. Perez EA, Romond EH, Suman VJ, Jeong JH, Sledge G, Geyer CE Jr, Martino S, Rastogi P, Gralow J, Swain SM, et al: Trastuzumab plus adjuvant chemotherapy for human epidermal growth factor receptor 2-positive breast cancer: Planned joint analysis of overall survival from NSABP B-31 and NCCTG N9831. J Clin Oncol 32: 3744-3752, 2014.

9. Criscitiello $C$ and Curigliano G: HER2 signaling pathway and trastuzumab cardiotoxicity. Future Oncol 9: 179-181, 2013.

10. Negro A, Brar BK and Lee KF: Essential roles of Her2/erbB2 in cardiac development and function. Recent Prog Horm Res 59: $1-12,2004$.

11. Bloom MW, Hamo CE, Cardinale D, Ky B, Nohria A, Baer L, Skopicki H, Lenihan DJ, Gheorghiade M, Lyon AR and Butler J: Cancer therapy-related cardiac dysfunction and heart failure: Part 1: Definitions, pathophysiology, risk factors and imaging. Circ Heart Fail 9: e002661, 2016.

12. Ezaz G, Long JB, Gross CP and Chen J: Risk prediction model for heart failure and cardiomyopathy after adjuvant trastuzumab therapy for breast cancer. J Am Heart Assoc 3: e000472, 2014.

13. Goldhirsch A, Gelber RD, Piccart-Gebhart MJ, de Azambuja E, Procter M, Suter TM, Jackisch C, Cameron D, Weber HA, Heinzmann D, et al: 2 years versus 1 year of adjuvant trastuzumab for HER2-positive breast cancer (HERA): An open-label, randomised controlled trial. Lancet 382: 1021-1028, 2013.

14. McKee PA, Castelli WP, McNamara PM and Kannel WB: The natural history of congestive heart failure: The framingham study. N Engl J Med 285: 1441-1446, 1971.

15. Seidman A, Hudis C, Pierri MK, Shak S, Paton V, Ashby M, Murphy M, Stewart SJ and Keefe D: Cardiac dysfunction in the trastuzumab clinical trials experience. J Clin Oncol 20: 1215-1221, 2002.
16. BrownSA, Sandhu Nand Herrmann J: Systems biology approaches to adverse drug effects: The example of cardio-oncology. Nat Rev Clin Oncol 12: 718-731, 2015.

17. Guglin M, Cutro R and Mishkin JD: Trastuzumab-induced cardiomyopathy. J Card Fail 14: 437-444, 2008.

18. Bowles EJ, Wellman R, Feigelson HS, Onitilo AA, Freedman AN, Delate T, Allen LA, Nekhlyudov L, Goddard KA, Davis RL, et al: Risk of heart failure in breast cancer patients after anthracycline and trastuzumab treatment: A retrospective cohort study. J Natl Cancer Inst 104: 1293-1305, 2012.

19. Smith LA, Cornelius VR, Plummer CJ, Levitt G, Verrill M, Canney $\mathrm{P}$ and Jones A: Cardiotoxicity of anthracycline agents for the treatment of cancer: Systematic review and meta-analysis of randomised controlled trials. BMC Cancer 10: 337, 2010.

20. Russell SD, Blackwell KL, Lawrence J, Pippen JE Jr, Roe MT, Wood F, Paton V, Holmgren E and Mahaffey KW: Independent adjudication of symptomatic heart failure with the use of doxorubicin and cyclophosphamide followed by trastuzumab adjuvant therapy: A combined review of cardiac data from the National Surgical Adjuvant breast and Bowel Project B-31 and the North Central Cancer Treatment Group N9831 clinical trials. J Clin Oncol 28: 3416-3421, 2010.

21. Yu AF, Yadav NU, Eaton AA, Lung BY, Thaler HT, Liu JE, Hudis CA, Dang CT and Steingart RM: Continuous trastuzumab therapy in breast cancer patients with asymptomatic left ventricular dysfunction. Oncologist 20: 1105-1110, 2015.

22. Martin M, Esteva FJ, Alba E, Khandheria B, Pérez-Isla L, García-Sáenz JA, Márquez A, Sengupta $\mathrm{P}$ and Zamorano J: Minimizing cardiotoxicity while optimizing treatment efficacy with trastuzumab: Review and expert recommendations. Oncologist 14: 1-11, 2009.

23. Hensley ML, Schuchter LM, Lindley C, Meropol NJ, Cohen GI, Broder G, Gradishar WJ, Green DM, Langdon RJ Jr, Mitchell RB, et al: American society of clinical oncology clinical practice guidelines for the use of chemotherapy and radiotherapy protectants. J Clin Oncol 17: 3333-3355, 1999.

24. Hering D, Faber L and Horstkotte D: Echocardiographic features of radiation-associated valvular disease. Am J Cardiol 92: 226-230, 2003

25. Tassan-Mangina S, Codorean D, Metivier M, Costa B, Himberlin C, Jouannaud C, Blaise AM, Elaerts J and Nazeyrollas P: Tissue doppler imaging and conventional echocardiography after anthracycline treatment in adults: Early and late alterations of left ventricular function during a prospective study. Eur J Echocardiogr 7: 141-146, 2006.

26. Oreto L, Todaro MC, Umland MM, Kramer C, Qamar R, Carerj S, Khandheria BK and Paterick TE: Use of echocardiography to evaluate the cardiac effects of therapies used in cancer treatment: What do we know? J Am Soc Echocardiogr 25: 1141-1152, 2012.

27. Ferté C, Massard C, Cohen A, Soria JC and Ederhy S: Trastuzumab-induced cardiotoxicity: Is it time for troponin for all patients? Am J Clin Oncol 35: 183-184, 2012.

28. Cardinale D, Colombo A, Torrisi R, Sandri MT, Civelli M, Salvatici M, Lamantia G, Colombo N, Cortinovis S, Dessanai MA, et al: Trastuzumab-induced cardiotoxicity: Clinical and prognostic implications of troponin i evaluation. J Clin Oncol 28: 3910-3916, 2010. 